\title{
Evaluation of lightning location and lightning current wave shape using measured lightning magnetic fields at two stations
}

\begin{abstract}
In this paper, an algorithm is proposed to evaluate a lightning location as well as the lightning current wave shapes at different heights along a channel using lightning measured magnetic fields at two stations. The proposed algorithm is based on the captured return stroke part of the magnetic fields directly in the time domain. The proposed method can support different engineering current models and can also be developed for different distances. The proposed method can be helpful for creating lightning location and lightning current data banks as it can estimate the full shape of the current as opposed to the usual methods used previously.
\end{abstract}

Keyword: Lightning location; Lightning magnetic fields; Return stroke current 